\title{
Sociolingüística. Una introducción epistémica y política
}

\author{
Ígor Rodríguez-Iglesias ${ }^{1}$ \\ Universidad Autónoma de Madrid, España
}

\begin{abstract}
Resumen
En este documento se presenta una panorámica general de la sociolingüística en torno a su fundación. Concretamente, se indican las condiciones sociales de producción del conocimiento sociolingüístico, esto es, la condición social de posibilidad a través de la cual se explica la profusión de estudios dedicados a la interacción discursiva a partir de un momento determinado y el interés por las variables sociales implicadas tanto en el uso específico de elementos lingüísticos determinados como en la comunicación interpersonal e intergrupal.
\end{abstract}

Palabras clave: sociolingüística, política, contexto social, poder.

\footnotetext{
$1 \quad$ Para correspondencia, dirigirse a: Ígor Rodríguez-Iglesias (igor.rodriguez@uam.es), Facultad de Filosofía y Letras, Departamento de Lingüística General, Lenguas Modernas, Lógica y Filosofía de la Ciencia, Teoría de la Literatura y Literatura Comparada, Campus de Cantoblanco, Universidad Autónoma de Madrid, 28049, Madrid, España. ORCID 00000003-3585-2724.
} 


\title{
SOCIOLINGUISTICS. AN EPISTEMIC AND POLITICAL INTRODUCTION
}

\begin{abstract}
In this document I present an overview of the sociolinguistics around its foundation. Specifically, I study the social conditions of sociolinguistic knowledge production, that is, the social condition of possibility through which a set of studies dedicated to discursive interaction emerges. Thus, I inquire into the interest in the social variables involved in the specific use of certain linguistic elements and in interpersonal and intergroup communication.
\end{abstract}

Keywords: sociolinguistics, politics, social context, power.

Recibido: 05/02/20

Aceptado: 28/12/20

\section{INTRODUCCIÓN}

Las condiciones sociales de producción del conocimiento -y, en general, del discurso y su circulación- son esenciales para comprender el surgimiento de eso que damos en llamar las disciplinas o las áreas de conocimiento. Nuevas perspectivas estas que, como aquellas contra las que reaccionan o a partir de las que surgen, están vinculadas a trayectorias intelectuales individuales e intradisciplinares, así como lugares de enunciación siempre situados (Haraway 1992), ya sea temporal (Wallerstein 1999) o simbólicamente (Castro-Gómez 2003; Dussel 2012; Grosfoguel 2008).

La sociolingüística no solo no escapa a esto, sino que, quizás más que ninguna otra perspectiva de estudio (junto con el Análisis Crítico del Discurso; Fairclough 1989; Wodak 1989), ha supuesto un giro, un giro discursivo, epistémico y metodológico (Pujolar 2012; Heller y McElhinny 2017), en consonancia con la mirada que, en diversas partes del mundo, conocerá la segunda mitad del siglo XX. Este artículo es una introducción a la sociolingüística, con un alejamiento epistémico de supuestos estructurales de la lengua, partiendo de la consolidación de estudios sociolingüísticos y discursivos no basados en lógicas gramaticales o estructurales, sino en las condiciones sociales de producción de los discursos y de su circulación, con una atenta mirada a las procesos sociopolíticos, especialmente, de carácter decolonial. 
Es por ello que partiré de breves apuntes sobre qué entendemos por sociolingüística, cómo esta no es un quehacer al margen del estudio lingüístico basado en el signo lingüístico y de qué modo la sociolingüística crítica supone un amplio estudio (y libertad de estudio), sin las acotaciones disciplinarias basadas en formas de pensar la realidad de modo reduccionista propio de un positivismo decimonónico que subyace a la forma de concebir los campos o áreas de estudios y disciplinas.

Para lograr este objetivo, no solo se ofrece esta mirada descriptiva sobre a qué se dedica la sociolingüística (crítica) de hoy y cómo surgió, sino qué causas políticas, sociales e históricas motivaron ese giro discursivo. Para ello, pongo a dialogar el nacimiento de la sociolingüística con las condiciones sociales y políticas que rodearon las circunstancias en torno a la década de 1960. Este objetivo lleva consigo mantener un constante paralelismo entre tales condiciones sociales, intelectuales y políticas de las personas investigadoras en el entonces nuevo campo de la sociolingüística con otros giros descolonizadores en el ámbito de las ciencias sociales. Entre otros, podemos señalar a una filosofía y sociología estrictamente caribeñas y latinoamericanas, como Eric Williams, Aimé Cesaire, Frantz Fanon, Sylvia Wynter, Óscar Varsavsky, Orlando Fals Borda, Enrique Dussel, Silvia Rivera Cusicanqui, entre otros y otras, que, si bien no tienen una influencia directa sobre los fundadores de la sociolingüística en EE.UU., sí sobre diversos grupos sociales descolonizadores al interior de ese país, que finalmente acabarán influyendo de algún modo sobre estos investigadores universitarios y de un modo muy decisivo - directa o indirectamente- sobre las generaciones futuras de diversas partes del mundo (Latinoamérica, Europa, etc.), especialmente actuales, con la irrupción de un giro decolonial en el ámbito de los estudios científico sociales, en general, y sociolingüísticos (y glotopolíticos), en particular.

En el quehacer que me ocupa en las páginas que siguen es importante además destacar el papel de las reinvindicaciones de los grupos latinos y negros oprimidos de los campus universitarios (qué pasaba fuera de las aulas y despachos, mientras se producían los textos académicos desde los departamentos) -acontecimientos no siempre narrados y, más bien, omitidos-, y diversos hechos revolucionarios en el sur global. Tomar en cuenta las resistencias y reacciones contra las opresiones en Latinoamérica, África y Asia y al interior de los Estados del norte global por los grupos constitutivos de tales sures simbólicos en lo que el caribeño F. Fanon llamó zonas del Ser (Fanon 1952) -es decir, zonas del No-Ser dentro de las Zonas del Ser-es muy importante para comprender el papel no solo de los pioneros y fundadores de la sociolingüística, sino de sus desarrollos en dos grandes 
líneas: la variacionista y la crítica, así como el papel de cada cual en el llamado mundo hispánico, que ocupa la parte final.

En lo que sigue, no se hace una lectura nacionalista de la realidad. Es decir, no se habla de cómo en España, en México o en Chile se llegó a hacer sociolingüística. $\mathrm{O}$, más generalmente, en Latinoamérica, frente a EE.UU., Canadá o Inglaterra. No es ese el objeto de este artículo. De lo que aquí se trata es de comprender cómo hemos heredado en las universidades una disciplina a partir de un punto de origen muy concreto, con una influencia específica sobre nuestras universidades latinoamericanas y españolas de EE.UU. (véase el trabajo de López Morales y la influencia de Labov, por ejemplo; o la raíz laboviana, incluyendo sus métodos, del variacionismo) y qué circunstancias sociales y epistémicas de nuestros sures influyeron sobre la expansión desde aquel norte y de sus desarrollos posteriores, con una mirada atenta a dos tipos de sociolingüística actuales, con gran influencia laboviana, una, y con aperturas, la otra, hacia críticas no solo eurocéntricas sino también descoloniales, de raíz latinoamericana (Dussel, CastroGómez, Rivera Cuscanqui) y afrocaribeña (Fanon, Wynter, Grosfoguel), afroamericana (Davis, Crenshaw), oceánica (Tuhiwai Smith) y africana (Oyèwùmi, Thiong'o). Es decir, que se trata también, en el contexto de los desarrollos de la sociolingüística, de ver cómo se ha incorporado a la misma el conocimiento crítico producido por y desde el sur global. Es de advertir que aquí sur y norte no son ejes geográficos, sino, al modo de Fanon, posicionalidades en las relaciones de poder, por lo que se identificará como sur global las opresiones y resistencias al interior de cada Norte global.

Téngase en cuenta que este es un artículo en cuyo título figura la palabra introducción, por lo que solo se intenta ofrecer un esbozo general, que debe ser abordado más detenidamente en sucesivas publicaciones futuras.

\section{EL AMPLIO CAMPO DE LA SOCIOLINGÜÍSTICA}

Los estudios lingüísticos ortodoxos basan parte de su conocimiento lingüístico explícita o implícitamente en torno a la noción de signo lingüístico, legitimando el quehacer académico del estudio de la lengua respecto de la fonética (sustancia de la expresión o del significante), fonología (forma de la expresión o significante), morfología (forma de la expresión o significante y forma del contenido o significado del signo lingüístico morfológico), sintaxis (forma de la expresión o significante y forma del contenido o significado del 
signo lingüístico oracional) y semántica (forma del contenido o significado de cada signo: morfológico, léxico y oracional). La sustancia del contenido (sentido, respecto de su forma: significado) bien puede ser relacionada con los tipos de contenido semánticos o niveles del significar, dado que estos se ocupan de lo comunicativo interaccional, en el caso del sentido y su denotación (Mill), referencia (Lyons) o designación real (Coseriu), así como de lo comunicativo no interaccional, en el caso del concepto (Baldinger, Heger) y la designación potencial (Coseriu), como designación del significado, estrictamente sistemático, abstracto y estático (Casas 2002; Rodríguez-Iglesias, 2018a). Este quehacer, propio de una semántica -especialmente estructural-, se ha ocupado del contexto (Coseriu 1967 y 1990) y de todas estas cuestiones de carácter referencial y representacional, donde estas cuestiones conforman una interfaz sociocognitiva que es también ámbito de las ideologías lingüísticas. Lo que estoy haciendo aquí no es supeditar mi estudio a la concepción del signo lingüístico, sino poniendo a dialogar la sustancia del mismo, como explicitud de contenido de carácter comunicativo, concreto y dinámico, las funciones del hablar, el esquema de la comunicación praguense y los tipos de contenido o niveles del significar con los estudios sociolingüísticos, que se han encargado de esta cuestión -rudimentariamente descrita por el estructuralismo en el famoso esquema de la comunicación de K. Bühler (1934), primero, y R. Jakobson (1968), después, de carácter conductista e inapropiado respecto de las relaciones de fuerza implicadas y otros aspectos en la comunicación interaccional, a pesar de ser la descripción privilegiada por el sistema escolar en sus libros de texto. Obsérvese que un tipo de sociolingüística, la variacionista, se ha dedicado a correlacionar las partes del signo lingüístico de cada nivel (especialmente su sustancia: variantes o sonidos, usos gramaticales, etc.) con una concepción de grupo social y una clasificación vertical de los grupos sociales específicas.

Si la sociolingüística se encarga, pues, de todo este amplio espectro de la sustancia del contenido y de la comunicación tanto interaccional como no interaccional (las representaciones sociales) y es, en general, el estudio del lenguaje con relación a su uso en sociedad (Yule 2007: 253), definición calcada de Hudson (1981: 11), la sociolingüística crítica, en concreto, centra su mirada en el hablar como estructuradora social y las ideologías lingüísticas que sustentan las prácticas discursivas, es decir, interaccionales $\mathrm{y}$, por tanto, sociales.

Así, la sociolingüística crítica se encarga de los siguientes aspectos: 1) del estudio de las prácticas lingüísticas como estructuradoras de las relaciones sociales; 2) del estudio de las prácticas lingüísticas como reproductoras o transformadoras de relaciones de poder y, específicamente, de desigualdad, con la lengua y otros elementos interseccionados, como marcador de 
dominación; y 3) del análisis de las ideologías lingüísticas, incluidas las que comportan los discursos de la ciencia lingüística o la filología. J. Pujolar ha desarrollado esta cuestión:

En termes generals, podem incloure dins la sociolingüística crítica els estudis que incideixen en almenys dos dels aspectes següents: 1) Es pregunten de quina manera les pràctiques lingüístiques incideixen en el funcionament de la societat, el constitueixen o ens ajuden a explicarlo. Per tant, no interessa de forma central la descripció dels fenòmens lingüístics com a tals. 2) Es pregunten de quina manera els usos lingüístics contribueixen a reproduir i a transformar les relacions socials i, especialment, les relacions de desigualtat entre grups socials definits per criteris de repertori lingüístic, classe social, gè- nere, sexualitat, edat, religió, raça o etnicitat. 3) Analitzen les ideologies lingüístiques, això és, les idees (sovint divergents) que persones i grups tenen sobre les llengües $i$ les pràctiques lingüístiques, com a expressions d'interessos sectorials i de determinades formes d'organització social i política. Això inclou sovint un qüestionament d'alguns pressupòsits bàsics de la lingüística com a disciplina, que tradicionalment ha tractat les llengües com a unitats independents caracteritzades per les seves estructures formals.

Per tant, tenim: 1) Les pràctiques lingüístiques com a fets socials. 2) Les pràctiques lingüístiques en els conflictes socials. 3) Les pràctiques lingüístiques com a representacions socials (Pujolar 2012: 5).

Monica Heller, Sari Pietikäinen y Joan Pujolar han publicado en enero de 2018 un manual práctico sobre metodología de etnografía sociolingüística crítica, bajo el título de Critical Sociolinguistic Research Methods. Studying Language Issues That Matter. Allí explicitan qué entienden por sociolingüística, por etnográfica y por crítica:

We use a critical ethnographic sociolinguistic approach.

-By critical we mean that we put questions of power and inequality in the centre of our inquiry. We ask what resources are important to whom, and how the social processes we examine have consequences. (Here our focus is mainly on consequences for people, but many scholars are also interested in consequences for organizations or communities, specific social practices, animal or environment). Second, a critical stance applies also to ourselves: we use our understandings about the role of language in social processes of power and inequality reflexively, that is, by applying them to how we undertake the social activity of doing research (as socially constructed and traversed by relations of power), and to how we make sense of the data we generate as a result of the research process. 
-By ethnographic we mean in-depth, situaded explorations of how these processes work and why, and what they mean to people and why (seeking to both describe and explain), using representative or telling cases to illustrate broader processes. By "situaded", we mean we attend to the specific conditions and contexts in which the processes we are interested in unfold.

-By sociolinguistic we mean two things. First, that we situate this book in a conversation about how language matters, although we think it can be useful to anyone undertaking critical ethnographic research (and which can draw on converging traditions that go by different names, notably linguistic anthropology, discourse analysis and some domains of applied linguistics). We are aware that sociolinguistics can mean different things to different people; here we opt for the term as an umbrela covering all kinds of investigations into how language matters, socially, politically and economically (Heller, Pietikäinen y Pujolar 2018: 2).

La frontera con el Análisis Crítico del Discurso (ACD) no es tajante y, por tanto, es difusa. La etnografía, el trabajar directamente con los y las hablantes y el relato de estas historias, es lo que distingue la sociolingüística crítica y las temáticas apuntadas de las que se ocupa.

El ACD estudia 'el lenguaje como práctica social' (Fairclough y Wodak 1997), y considera que el contexto de uso del lenguaje es crucial (Wodak 2000c; Benke 2000). Además, el ACD se interesa de modo particular por la relación entre el lenguaje y el poder. De modo más específico, el término ACD se utiliza hoy en día para referencia al enfoque que, desde la lingüística crítica, hacen los académicos que consideran que la amplia unidad del texto discursivo es la unidad básica de comunicación. Esta investigación tiene en cuenta, de modo muy concreto, los discursos institucionales, políticos, de género y mediáticos (en el más amplio sentido) que dan testimonio de la existencia de unas más o menos abiertas relaciones de lucha y conflicto" (Wodak 2003: 21).

Los discursos son estudiados por su contenido. Es una diferencia importante con respecto a lo que llamamos habitualmente Análisis del Discurso (AD), que más propiamente podríamos denominar Análisis Lingüístico del Discurso, ya que sus objetivos no son tanto lo que se dice, sino cómo se dice, grosso modo: la sustancia y la forma del expresión (y su correlación de contenido) frente a la forma y la sustancia del contenido, su referencialidad y los agentes sociales implicados en ello, amén de significados sociales y consecuencias tanto comunicativas como ontológicas.

Si desde la década de 1970 el Análisis del Discurso se ha venido ocupando de "las categorías analíticas y las nociones, propias del discurso, como 
cohesión, coherencia, macroestructuras, superestructuras y otras estructuras convencionales de los géneros discursivos [...] cada vez más independientes de la lingüística y de la gramática oracional" (a diferencia de los primeros desarrollos de la gramática del texto de la década anterior) (van Dijk 2015: 17-18), el Análisis Crítico del Discurso explora "nociones, tan diversas pero relacionadas, de poder, ideología, contexto y conocimiento, y sus aplicaciones a los estudios del racismo discursivo, sobre todo en el discurso de las élites simbólicas: el discurso político, de la prensa y de los libros de texto" (ibid., p. 24), al menos en lo que respecta al trabajo de Teun van Dijk, que ha propuesto el cambio de nombre de ACD a Estudios Críticos del Discurso, pues análisis aún es deudor de una concepción estructural y sus respectivas indagaciones: análisis fonológico, morfológico, sintáctico, semántico. Se trata de estudiar contenido y sus efectos, no de analizar unidades discretas, segmentales, sistemáticas, abstractas y estáticas, como en la concepción lingüística estructuralista.

Aunque actualmente se ha adoptado de manera generalizada la fórmula Análisis Crítico del Discurso (ACD), me gustaría proponer que se la cambie por Estudios Críticos del Discurso (ECD) por una cantidad de razones obvias. La razón principal es que los Estudios Críticos del Discurso no son-como con mucha frecuencia se supone, especialmente en el campo de las ciencias sociales- un método de análisis del discurso. No existe un método semejante. Los ECD utilizan cualquier método que sea pertinente para los objetivos de sus proyectos de investigación y esos métodos son, en gran medida, los que se utilizan generalmente en los estudios del discurso (Dijk 2009: 21).

Y lo mismo apunta Dijk para el Análisis del Discurso, que pasa a denominar Estudios del Discurso (ES): "por la misma razón, [...] no es un método, sino antes bien una esfera de la práctica académica, un cruce de disciplinas distribuido a través de todas las humanidades y las ciencias sociales" (ibid.).

Norman Fairclough ya había definido en 1989 los estudios críticos en lingüística como "critical language study" (CLS), donde igualmente el objetivo es el estudio del poder y la ideología con relación a la lengua:

The approach to language which will be adopted here will be called critical language study, or CLS for short. Critical is used in special sense of aiming to show up connections which may be hidden from people such as the connections between language, power and ideology referred to above. CLS analyses social interactions in a way which focuses upon their linguistic elements, and which sets out to show up their generally hidden determinants in the system of social relationships, as well as hidden effects they may have upon that system (Fairclough 1989: 5). 
En la última parte de este artículo, vamos a ver cómo estos desarrollos críticos van a incorporar, como se ha avanzado en la introducción, conocimiento crítico desarrollado en las subalternidades latinoamericanas y norteamericanas. En la siguiente sección, se avanzan algunas de estas autorías, como contexto social.

\section{CONDICIONES SOCIALES PARA UNA NUEVA PRODUCCIÓN DE CONOCIMIENTO}

Se puede decir que esta década de 1980 es la que va a suponer el punto de inflexión en las ciencias del lenguaje con perspectiva crítica. De hecho, según van Dijk (2009: 11), el acta fundacional, por así decirlo, de los ECD es la publicación en 1979 del libro Language and Control, de Robert Fowler, Bob Hodge, Gunther Kress y Tony Trew. Además del libro de Fairclough de 1989, merece igual mención el de Ruth Wodak como editora: Language, Power and Ideology: Studies in Political Discourse (1989), así como el del propio Fowler de 1991, Language in the news. Discourse and ideology in the press.

Este ambiente crítico en las ciencias del lenguaje tiene precedentes claros en la filosofía y la sociología críticas. En la primera, destaca, de un lado, Michel Foucault, con su orden del discurso (1970) y otros tantos análisis sobre la arqueología del saber (1979) y el castigo (Vigilar y castigar 1975), para entender la conformación disciplinar y el poder, durante la década de 1970. En la sociología crítica (del lenguaje, de la educación, de la cultura) destaca Pierre Bourdieu. Este desde 1975 a 1982 publicó diversos artículos recogidos en un libro fundamental: ¿Qué significar hablar? Economía de los intercambios lingüísticos (1982[2008]). Un año antes, también en Francia, Louis-Jean Calvet publicaba Lingüistica y colonialismo. Breve tratado de glotofagia (1972).

En paralelo, la filosofía de la liberación de E. Dussel, desde las décadas de 1960 y 1970 en adelante, explicitada en un libro homónimo (1983 y 1996), escrito en el exilio, en México, tras un atentado con bomba en su casa por parte de los fascistas, en Argentina, hablaba del mismo oprimido del brasileño Paulo Freire, de los mismos condenados de la Tierra que el martinicano Frantz Fanon (1961), cuya denuncia en Piel negra, máscaras blancas (1952) de un mundo patológicamente racista dividido en el Ser y el no-Ser fundamenta la crítica decolonial, dentro de la sociolingüística (Codó, 
Patiño y Unamuno 2012; Rodríguez-Iglesias 2016 y 2018b) y fuera de ella (Grosfoguel 2013; Maldonado-Torres 2015).

Durante este periodo de la llamada Guerra Fría, diversas voces desde Latinoamérica hablan ya de "ciencia guerrillera" y "científico rebelde" (Varsavsky 1969 apud Fals Borda 1987: 16) y "ciencia rebelde y subversiva" (Fals Borda 1987: 16), que "entroniza la crítica inteligente, batalla contra el colonialismo en todas sus formas [...] y estimula la formación de frentes interdisciplinarios" (ibid.).

Es el tiempo de pensadoras y pensadores del Caribe y Latinoamérica de gran calado intelectual: el discurso sobre el colonialismo de Aimé Césaire (1950), los ensayos decoloniales de Sylvia Winter (décadas de 1960 y 1970; en realidad, hasta hoy), la otra mirada a la historia (que el eurocentrismo ni contaba entonces ni cuenta hoy) del guyanés Walter Rodney (1972), entre otros, incluyendo al propio Fanon (1952). Este tiempo es el de Rosa Parks, The Black Panthers, Martin Luther King y Malcolm X. Es el tiempo de Combahee River Collective y la National Black Feminist Organization. Y el de Women Strike for Peace. Es el tiempo de Simone Beauvoir, Betty Friedan, National Organization for Women o Kate Millet. El de la Revolución cubana (1959 en adelante). El de Mayo del 68 en Francia. El del Third World Liberation Front (TWLF) y la larga huelga estudiantil en 1969 en la Universidad de California y otras universidades en Texas y el sur de California, reprimidas por la Guardia Nacional, con hasta 4.000 arrestos y el asesinato a manos de la policía de estudiantes negros (Schwendinger, Schwendinger y Lynch 2002: 51; cf. Grosfoguel 2007: 33). Es un tiempo, pues, convulso, de disputa de espacios, de luchas por ocupar el espacio de la palabra y de la irrupción de los cuerpos coloniales y colonizados en el terreno de la misma; de represión y de guerra también: guerra de Vietnam y Camboya (década de 1960), intervención de la CIA en Centroamérica y el Cono Sur con el establecimiento de dictaduras militares (décadas de 1960 y 1970), bloqueo de Cuba, Guerra Fría, etc. Es el tiempo de los tratados de París o CECA (1951) y Roma (1957), bases fundacionales de la futura Unión Europea, que permite vislumbrar la geopolítica venidera. Este es el tiempo de la segunda escuela sociológica de Chicago, que en la década de 1950 centrará su mirada en el interaccionismo simbólico (una figura importante acá es Erving Goffman), y, en el mismo ámbito geográfico de los primeros congresos de sociolingüística, la escuela sociológica de Palo Alto, cuya premisa fundamental es que "communication is the matrix in which all human activities are embedded" (Ruesch 1951: 13). En paralelo, en Alemania, el paso de la primera escuela de Frankfurt a la segunda, escuela de pensamiento filosófico crítico, con J. Habermas y K.-O. Apel, como representantes paradigmáticos. 
Es en este tiempo en el que nace y se desarrolla la sociolingüística. La condición de posibilidad de tomar en cuenta a quien habla, a los grupos sociales, a las comunidades de habla. Los desarrollos de la Estudios del Discurso (Análisis del Discurso) en la década de 1960 están supeditados a la lingüística, como la exploración de un nivel de indagación más allá de la oración, esto es, el texto (tal y como hemos visto, más arriba, con T. van Dijk).

Es en esta década en que la sociolingüística ocupará un espacio que no podía ser habitado por la lingüística estructural, ya estructuralista, ya generativista (y mucho menos los estudios normativistas de las filologías). Es a lo que apunta Bernard Spolsky cuando dice que quienes serán los fundadores de la sociolingüística "deal with the evolutionary inadequacies of human physiology, rejecting the ideology established in mainstream linguistics by Chomsky's lack of interest in meaning and his focus on the competence of an 'idealized monolingual"' (Spolsky 2011: 5). En estas fechas, en EE.UU., el generativismo acaba de nacer. En 1957 y 1965, Noam Chomsky está publicando los libros fundacionales de la lingüística que dominará EE.UU., el generativismo: Syntantic Structures y, especialmente, Aspects of Theory of Syntax, respectivamente, en respuesta a los planteamientos distribucionales de Z. Harris y, en concreto, de constituyentes inmediatos de R. Wells. La fecha fundacional de la sociolingüística es relevante, dado el contexto: la emergencia generativista, los desarrollos funcionalistas de la Segunda Escuela de Praga, con František Daneš y Jan Firbas, especialmente; y toda la convulsión social aludida. Es un tiempo de cambio, de giro en general y, en concreto, de giro discursivo (Pujolar 2012). A diferencia de Spolsky, Heller y McElnny apuntarán que esta nueva área de estudios, la sociolingüística, se postulará "in order to engage directly with the influential claims of generativism, widely heralded as nothing short of a revolution" (Heller y McElhinny 2017: 195).

Las décadas de 1950 y 1960 conocen un incremento del interés general en las relaciones de la lengua en su uso social. Tal y como señalan Heller y McElnny,

[t]he central set of actors in the emergence of sociolinguistics mainly belonged to the generation born between the wars, coming of age in wartime or the postwar period. Many influential scholars were of working-class or regional having directly experienced anti-Semitism in Nazi Europe. Many had been soldiers or occupied other military roles during or after Worl War II or in the Korean War (for Americans) or the Algerian war (for French sociolinguists). Some grew up in peripheral and colonial contexts (such as Tunisia or Corsica). Some became involved in international or domestic welfare state development projects, working among colonial peripheral groups, in urban working-class 
neighbourhoods, or, in the long-stansing Americanist tradition, on Native American reserves" (Heller y McElhinny 2017: 196).

Por tanto, estos sociolingüistas habían desarrollado previamente una sensibilidad diferente a los temas de carácter, ante los problemas donde la lengua juega un papel fundamental: desde situaciones de bilingüismo y contacto y conflicto lingüístico hasta el acceso a los recursos lingüísticos, a través del sistema educativo u otras instituciones sociales, y cómo estos accesos y repertorios eran disputados.

\section{ORÍGENES DE LA SOCIOLINGÜÍSTICA}

El profesor Mauro Fernández (1997) -basado en Konrad Koerner (1986; 1991), Roger Shuy (1989, 1990), Wilfred Lehman (1981), Yakob Malkiel (1976), Stephen Murray (1983, 1994) y el Handbook of Sociolinguistics editado por Ulrich Ammon (1987-88) - ha mostrado una breve historia de los orígenes de la sociolingüística, que nos sirve aquí como panorámica general de precedentes y conformación de la disciplina que nos concierne, en el ámbito de las Ciencias del Lenguaje.

Wilfred Lehman (1981 apud Fernández 1996) adelanta el nacimiento de la sociolingüística a 1949, con Haver C. Currie (1949) "The Relationship of Sociolinguistics: The Relationship of Speech to Social Status". Por su parte, Yacob Malkiel (1976 apud Fernández 1997) fecha el inicio de la sociolingüística a partir de la aparición de diversas publicaciones relevantes:

- Weireich, Uriel (1953). Language in Contact. Findings and Problems. The Hague/Paris/ New York: Mouton.

- Haugen, Einar (1953). The Norwegian Language in America: A study in bilingual behavior, by Einar Haugen, Philapelphia, University of Pennsylvania Press, 2 vols.

- Anthropology Today (1953).

- Sommerfelt, Alf (1953). "Language, society and culture". Summer seminary. University of Michigan.

- Haugen, Einar (1954).

- Columbia's Linguistics Today (1954).

- Weinreich, Uriel (1955). Review of The Norwegian Language in America: A study in bilingual behavior, by Einar Haugen, 
Philapelphia, University of Pennsylvania Press, 2 vols., 1953. Word 11, pp. 165-168.

- Y el surgimiento de un nuevo interés por Edward Sapir.

Mauro Fernández (1997) añade más precedentes en estas fechas:

- Antoine Meillet.

- Fishman, Joshua (1950). Curso de Sociology of language, en Yeshiva University, Nueva York.

- Ferguson, Charles Albert (1959). "Diglossia”, Word 15, pp. 325-340.

- Ferguson, Ch. A. y Gumperz, John (1960). Linguistic Diversity in South Asia (International Journal of American Linguistics 26, 3, 2). Bloomington: Indiana University Press.

- Hertzler, J. O. (1953). "Toward a Sociology of Language”. Social Forces 32(2), pp. 109-119.

- Cohen, Marcel (1956). Pour une sociologie du langage.

Al tiempo, encontramos diversos antecedentes en la lingüística sincrónica desde siglo XIX:

- Tradición sociológica: J. A. Schmeller, K.M. Rapp, K. Weinhold, P. Wegener.

- Geografía lingüística: G. Wenker, J.L. Gilliéron, E. Edmont, J. Judd, K. Jaberg, P. Scheuermeier, G. Rohlfs, M. L. Wagner, H. Kurath.

- Precedentes por su reacción contra el biologicismo de Schleicher: W. D. Whiney, H. Stheinthal, M. Bréal, H. Paul, J. Badouin de Courtenay.

A esto podemos añadir (apud Fernández 1997) los usos previos de denominaciones parecidas a la sociolingüística o tratamientos similares:

- 1821. Schmeller, Johann Andreas. Sprachsoziologie.

- 1896. Fundación del Année sociologique. Sección dedicada al lenguaje en su primer número. No obstante, he revisado estos números y lo primero que he visto ha sido, en el número de 1904, el artículo de Meillet sobre el cambio de las palabras.

Además, las preocupaciones por la dimensión social del lenguaje, a lo largo de la historia son conocidas: por ejemplo, Panini (Kiparsky 1979 apud Fernández 1997), Fernão de Oliveira (Coseriu 1981 apud Fernández 1997), por no hablar de los gramáticos, ortógrafos y autores de diálogos, cuya 
preocupación social sobre la lengua fue más bien antisocial (véase Cantera Ortiz de Urbina 1992; Rodríguez-Iglesias 2018).

W. Labov (1983), al ocuparse del estudio del cambio lingüístico en su contexto social, realiza un recorrido historiográfico sobre las ideas lingüísticas en torno a si es un hecho individual o social el lenguaje: "Los lingüistas parecen, por tanto, dividirse en dos grandes grupos respecto a este problema" (1983: 330). Puede ser resumido en el siguiente cuadro, de elaboración propia a partir del texto de Labov (1983: 326 y ss.), en orden cronológico y respecto de la influencia que los lingüistas del siglo XIX W. D. Whitney y H. Paul ejercen sobre los lingüistas del siglo XX:

\begin{tabular}{|c|c|}
\hline Hecho social & Hecho individual o asocial \\
\hline W. D. Whitney & H. Paul \\
\hline H. Schuchardt & J. Sweet \\
\hline A. Meillet & $\begin{array}{c}\text { Escuela de Praga (N. } \\
\text { S. Trubetzkoy, etc.) }\end{array}$ \\
\hline J. Vendryes & A. Martinet \\
\hline O. Jespersen & L. Bloomfield \\
\hline Sturtevant & N. Chomsky \\
\hline--- & M. Halle \\
\hline--- & Kurytowicz \\
\hline
\end{tabular}

La preponderancia de los lingüistas "individualistas" es clara, a la luz de los acontecimientos y rigen las grandes líneas de investigación e indagación: estructuralismo, generativismo y tradicionalismo (RAE y similares). Sin embargo, como señala Labov:

El eclipse de los lingüistas sociales se debe primordialmente a las limitaciones de sus propios trabajos y escritos sobre el contexto social del lenguaje. Tenían la limitación de ser apenas una explicación intuitiva de unos pocos acontecimientos anecdóticos extraídos de sus conocimientos generales. Cuando leemos los comentarios de Whitney, Meillet, Jespersen o Sturtevant no podemos decir que ninguno de ellos conocía más cosas acerca del impacto de la sociedad sobre el lenguaje que cualquier otro lingüista; sencillamente hablaban más del tema (Labov 1983: 334-335).

El desconocimiento general sobre teorías sociológicas, del que habla Labov, lleva a uno de los fundadores de la sociolingüística, Dell Hymes, a parafrasear a Edward Sapir: el primero habla de "why linguistics needs the sociologist" 
(Hymes 1967) y el segundo, de "why Cultural Anthropology Needs the Psychiatrist" (Sapir 1938). Ambos enfatizan el carácter social del fenómeno lingüístico, explicitada en la frase de Sapir, que también cita Hymes, "we cannot thoroughly understand the dynamics of culture, of society, of history, without sooner or later taking account of the actual interrelationships of human beings" (Sapir 1938: 11).

Con la presencia de Hymes y otros, el curso académico 1963-1964 (y ese verano) (Fernández 1997; también Borrego Nieto 1981) ha sido definida como la fecha fundacional de la sociolingüística. En este punto, procede advertir que en esta época hay fluctuaciones en el nombre de la disciplina entre Sociolinguistics (Labov) y Sociology of Language (Fishman), cuyos límites han sido precisados por algunas autorías, pero sin consenso general (Romaine 1994; Labov 1983; Fernández 1997), aunque el nombre más usado es el de sociolingüística. Un esquema, a continuación, nos permite ver un resumen cronológico de ese curso y, por tanto, de las actas fundacionales de la sociolingüística en EE.UU.:

$\cdot 1963$

- Congreso sobre Etnografía de la Comunicación.

- Noviembre.

- En San Francisco.

- John Gumperz y Dell Hymes (1964). The Ethnography of Communication. Publicación de los trabajos de este congreso.

$\cdot 1964$

$\circ$ Congreso.

- 11-13 mayo.

- En Los Ángeles: Center of Conferences Lake Arrowheads, University of California at Los Angeles.

- Organizado por el Center for Research in Language and Linguistics, de esa universidad.

- Brigth, William (1966). Sociolinguistics. Publicación de los trabajos de este congreso.

- Participantes:

- De la University of California at Los Angeles:

- Birnbaum, Henrick.

- Brigth, William.

- Bryan, Margaret.

- Dillon, Myles (también pertenecía al Institut for Advanced Studies, de Dublín). 
- Garfinkel, Harold.

- Ivić, Milka (también Universidad de Novi Sad, antigua Yugoslavia).

- Ivić, Pavle (también Universidad de Novi Sad, antigua Yugoslavia).

- Law, Horward W.

- Mathiot, Madeleine.

- Richardson, Irvine (también Michigan State University).

- Stockwell, Robert P.

- Invitados:

- Ferguson, Ch. A. (Center for Applied Linguistic, Washington).

- Fischer, John L. (Tulane University, Nueva Orleans).

- Friedrich, Paul (University of Chicago).

- Garvin, Paul L. (Bunker-Ramo Corporation, California).

- Gumperz, John J. (University of California at Berkeley).

- Haugen, Einar(Harvard University, Massachusetts).

- Hoenigswald, Henry (University of Pennsylvania).

- Hymes, Dell (University of California at Berkeley).

- Kelley, Gerald (Cornell Univesity, Nueva York).

- Labov, William (Columbia University, Nueva York).

- Mc David, Raven I. (University of Chicago).

- Paper, Herbert (Univesity of Michigan)

- Samarin, William J. (Hartford Seminary Foundation, Connecticut).

- Sjoberg, Andrée J. (University of Texas).

Es obvio que la fundación de unárea de estudios no se reduce solo a las personas presentes en un par de reuniones científicas, pero tales acontecimientos son más que simbólicos por la presencia y protagonismo de figuras altamente destacadas en los desarrollos posteriores de la sociolingüística. No obstante, se deben considerar otras figuras que, posteriormente, serán influyentes, aunque su trabajo en estos momentos fuera periférico, precisamente por las mismas condiciones que denunciaban en esos momentos o denunciaron luego. Es destacable el protagonismo de mujeres que pondrán su atención precisamente en el género como categoría de análisis. Destacan, en este 
sentido, de un lado, Susan Philips, Susan Ervin-Tripp y Gillian Sankoff y, de otro, Robin Lakoff, Deborah Tannen y Judith Butler. Grosso modo, en todas ellas juega un papel importante John Gumperz. Es en las últimas donde desarrollos enfocados al feminismo serán determinantes en generaciones siguientes. Las cuestiones relativas al género y a las relaciones de dominación se convertirán en centrales en estas investigadoras.

Por otro lado, en lo que respecta al mundo hispánico, tal y como expresan Codó, Patiño Santos y Unamuno (2012),

la mayor parte de los estudios en sociolingüística en Hispanoamérica están marcados por una tradición objetivista como consecuencia de cuestiones tanto epistemológicas como históricas e ideológicas. Así, por un lado, no se puede olvidar el impacto que tuvieron entre los sociolingüistas los estudios de tipo cuantitativo-correlacional, inspirados en los trabajos variacionistas de Labov (1972), de figuras tan reconocidas como Humberto López Morales (1983) en Puerto Rico o Beatriz Lavandera (1984) en Argentina (Codó, Patiño Santos y Unamuno, 2012).

La fundación anglosajona de la sociolingüística tiene en Latinoamérica y en España un paralelismo diferente, una preocupación por los estudios dialectológicos relacionada con una emancipación americana de la península ibérica más allá de las independencias formales, como procesos de legitimación de las realidades nacionales criollas (es decir, sin el reconocimiento de la diversidad étnica y plurinacional, que más tarde se ha constituido en problema de estudio) (Del Valle y Stheman 2004), lo que se une a las dictaduras fascistas latinoamericanas y española y otros procesos, en el contexto de las democracias liberales burguesas, de "políticas de homogeneización étnica y lingüística llevados a cabo durante el siglo XX en América del Sur -a costa de sus pueblos orginarios y a costa de las poblaciones inmigrantes", que "conllevaron la imposición de leyes reguladoras sobre el uso único o prioritario de la lengua nacional" (Codó, Patiño Santos y Unamuno, 2012), panorama que comienza a cambiar desde las décadas de 1980 y 1990 en adelante (íbid.).

Esta preocupación implicó la profusión de estudios en Latinoamérica en estos sentidos ( $c f$. Codó, Patiño Santos y Unamuno, 2012):

- Estudios dialectológicos:

- Lope Blanch (1974) sobre el español de México.

- Fontanella de Weinberg (1976) sobre el español de Argentina.

- Montes Giraldo (1977) sobre el español de Colombia.

- Luis Flórez (1958-1983), con el Atlas Lingüístico y Etnográfico de Colombia. 
- Estudios de contacto lingüístico:

- Granda (1978) sobre africanismos en español.

- Rona (1965) sobre variedades fronterizas en América del Sur.

- Morínigo (1965) y Rubín (1974) sobre indigenismos.

- Política lingüística:

- Arnoux (1999).

- Hamel (2008).

- Tipología lingüística de lenguas de los pueblos originarios:

○ Fernández Garay (1995).

La comparación de ambos esquemas nos da una perspectiva sobre los orígenes de la sociolingüística y qué sucedía, en general, en Latinoamérica y España, atrapadas por procesos dictatoriales y dinámicas históricas de consolidación nacional (incluida España, tras lo que en este país se llamó -y se sigue llamando en sus libros de texto de historia de la secundaria- "el desastre de 1898", en referencia a la definitiva disolución de su Imperio). La influencia sobre los desarrollos de los estudios lingüísticos es clara a partir de la década de 1980. Estos estudios lograrán subirse al carro de la sociolingüística, en el sentido en que se desarrollaba en EE.UU., Canadá y Reino Unido, con una perspectiva variacionista (laboviana), cuyos desarrollos la hispanística reinterpretará en términos clasistas, con un abuso del término laboviano, de origen weberiano, prestigio, en la actualidad enfrentado con el de privilegio ( $c f$. Piller 2016; Rodríguez-Iglesias 2016). La línea abierta por Gumperz y Hymes llegará más tarde: grosso modo, en el siglo XXI, con desarrollos bastante importantes.

\section{DOS GRANDES LÍNEAS}

Estos y estas sociolingüistas proceden de tradiciones o disciplinas diferentes: principalmente, la antropología y la lingüística. Pronto se ven las diferencias de criterios, que quedan bien patentes en dos líneas: la representada por William Labov, de corte más cercano a la lingüística estructuralista y al que, por tanto, le sobrevive la dicotomía lengua/habla de Gabelentz (1891) y Saussure (1916) ( $c f$. Blommaert 2010: 4), y la representada por John Gumperz y Dell Hymes en California y por Joshua Fishman en Nueva York, enriquecidos por aproximaciones más amplias, en tanto que conocedoras de lo social, como la antropología y la psicología social, respectivamente. 
Precisamente, una crítica que hace T. van Dijk (2015) a los estudios del texto de la década de 1970, de los que él es fundador, es la falta de atención al contexto, esto es, a la realidad misma en la que tienen lugar las interacciones comunicativas, humanas, sociales.

Si comparamos estos enfoques surgidos en el marco de la lingüística con la etnografía del habla de Dell Hymes, cabe destacar la falta de la contextualización social y cultural de esas 'gramáticas'. Básicamente, se trataba ante todo de una extensión del objeto de descripción desde oraciones a textos enteros $-\mathrm{e}$, inicialmente, recurriendo a las mismas categorías creadas por la gramática estructural o generativa para el estudio de las oraciones. Las categorías analíticas y las nociones, propias del discurso, como cohesión, coherencia, macroestructuras, superestructuras y otras estructuras convencionales de los géneros discursivos aparecen solamente en los estudios del discurso a partir de los años 70, y de forma cada vez más independientes de la lingüística y de la gramática oracional (Dijk 2015: 18).

W. Labov es el que va a tener una mayor repercusión en la hispanística, especialmente, gracias a que su obra The social stratification of English in New York City (Labov 1966), fundamentada en su trabajo de campo llevado a cabo en Nueva York, va a influir en parte en el libro sociolingüística de Humberto López Morales (1989), cuyo estudio homólogo se desarrolla en San Juan de Puerto Rico. Así mismo, esta línea dará lugar a trabajos sociolingüísticos de carácter estructuralista, basados en métodos cuantitativos y estadísticos de recolección de datos y en una concepción variacionista de inspiración tanto laboviana como couseriana, que, además, seguirán lo ya iniciado desde la geografía lingüística y la dialectología, como en Manuel Alvar o Gregorio Salvador, o más recientemente Francisco Moreno Fernández o Luis Escoriza.

Gumperz, Hymes y Fishman influirán en otros sociolingüistas, que seguirán otros derroteros muy diferentes, de carácter discursivista, interaccional, situado y con concepciones o influencias, además de gumperzianas, procedentes de Michel Foucault y Pierre Bourdieu, y que entran en España gracias a Luisa Martín Rojo, Celso Caccamo, Helena Calsamiglia y Amparo Tuson, entre otras, de una generación rebelde y fundamental para quienes hemos sido su alumnado, ya que sus experiencias predoctorales y posdoctorales supusieron una modernización, sin lugar a dudas, de una filología obsoleta y anclada en un nacionalismo españolista propio del franquismo.

Esas dos grandes líneas ponen de relieve cómo desde el inicio son patentes las ramificaciones y amplitud de temas de la sociolingüística, ligada 
esta diversidad a la formación e intereses diferentes de cada sociolingüista. Esta variedad de líneas se mantiene hoy en día y es, a la luz de la historia de eso que llamamos la disciplina, consustancial a la misma. Así lo indican tanto Jan Blommaert: "Like every disciplinary label, 'sociolinguistics' covers a tremendous variety of approaches" (Blommaert 2014: 131), como Monica Heller, Sari Pietikäinen y Joan Pujolar, quienes desde el mismo amplio campo reconocen la diversidad de puntos de vista sobre qué es la sociolingüística y, bajo esta etiqueta, sus alcances e intereses investigativos, "how language matters, socially, politically and economically" (2018: 2). Esta misma diversidad es patente en la lingüística estructural, la psicología, la sociología, etc.

Estas dos grandes líneas de la sociolingüística son, prácticamente, irreconciliables o, dicho de un modo menos tremendista: cada línea viene conducida por una pregunta de investigación diferente, que exige métodos diferentes (cuantificar o no cuantificar, etc.). La primera, la variacionista, está supeditada a la interpretación epistémica del estructuralismo sobre qué son y cómo funcionan (eso que llamamos) las lenguas. No obstante, el propio Labov reconoce que fuera de la sociedad no es posible hacer lingüística, criticando que "la gran mayoría de los lingüistas se han dedicado resueltamente a la contemplación de sus propios idiolectos" (Labov 1983: 23 ), por lo que ha defendido que "el conocimiento intersubjetivo en lingüística debe hallarse en el lenguaje hablando tal como lo utilizan en la vida cotidiana los miembros de una sociedad organizada" (Labov 1983: 23). Sin embargo, a la luz de su quehacer y de los procederes similares (vid. Carbonero Cano 2003; García Marcos 2015), este tipo de estudios correlacionan las unidades que conforman el producto lingüístico (muy especialmente, fonética, morfonológica y léxicamente) con variables de carácter social de los hablantes estudiados como sujetos informantes, tales como clase social, sexo, edad, profesión, nivel de estudios, etc., concibiendo la variación sociolingüística en términos de diatopía y diastratía (en terminología de Flydal 1951) o diafasía (en terminología de Coseriu 1957) (cf. Coseriu 1999: 298), amén de variación diasexual, diageneracional, etc. (Casas Gómez y Escoriza Morera 2009; Rodríguez-Iglesias 2013).

Uno de los problemas de este proceder es la no problematización de los instrumentos conceptuales, especialmente, los sociológicos: qué es clase social, qué es sexo o qué es situación comunicativa, sometidos a continuas revisiones y discusiones al interior de los estudios de donde son tomados ( $c f$. Bourdieu 2008; Block 2013). Es decir, la falta de reflexividad metodológica de la que hablan Pérez-Milans (2012) y Codó, Patiño y Unamuno (2012).

Por ejemplo, en lo concerniente a la variable sexo, Duranti (2000), a partir de Susan Gal (1989), expone: 
El conocimiento de la clase social de los participantes, de la historia familiar, del género, nos proporciona tan solo una parte -aunque virtualmente importante- de la historia que se está construyendo. Como ha indicado Susan Gal (1989), los recientes trabajos sobre el lenguaje de las mujeres rechazan de raíz cualquier idealización esencialista de "una voz de mujer" y de la noción implícita de una cultura separada, y avanzan la hipótesis de "prácticas lingüísticas más contradictorias y ambiguas, que difieren de mujeres de unos grupos étnicos y clases a otras, en un aspecto que va de la adaptación a la oposición, la subversión, el rechazo o la reconstrucción de definiciones culturales hegemónicas" (Gal 1989: 4). Si queremos hablar de género, habla y poder, sostiene Gal, lo primero que necesitamos hacer es averiguar qué es lo que se entiende por poder y discurso de poder en las distintas culturas. Para los antropólogos lingüísticos, una noción de poder diferenciada significa aceptar que posiblemente encontraremos prácticas lingüísticas distribuidas de modos distintos en función de las coordenadas de género, clase o etnia (Duranti 2000: 29-30).

Poder y acción son dos cuestiones fundamentales en la sociolingüística crítica, específicamente, en el sentido de Foucault, a partir de cómo se constituye en las prácticas cotidianas. En otro lugar (Rodríguez-Iglesias 2017), he correlacionado el poder foucaultiano con la matriz de dominación de la feminista negra norteamericana Patricia Hill Collins (2000), así como autorías decoloniales han correlacionado al mismo Foucault (2003), con relación a su análisis de la biopolítica de dominación, con otros planteamientos más abarcadores desde el punto de vista ontológico, al partir desde otras experiencias, no estando en contradicción con su crítica a esta división internacional del ser humano sobre cuestiones tan intrínsecamente personales e ineludibles, como el sexo o el origen geográfico, pero que amplían sus alcances y, desde luego, fueron formuladas antes, como la epidermización de Fanon (Grosfoguel 2018: 18) o la pregunta de Soujourner Truth a las feministas blancas burguesas: “¿Acaso yo no soy una mujer?" (Rodríguez-Iglesias 2017). La reflexividad metodológica es, pues, fundamental. La continua problematización, en definitiva, ya que nos permite recorrer caminos al poner sobre la mesa las limitaciones del propio investigador o investigadora, de sus herramientas y del estado de conocimiento de su tiempo y su lugar de enunciación.

Desde el ámbito sociolingüístico, José del Valle ha criticado recientemente que

la sociolingüística variacionista es profundamente descriptiva, está todavía anclada en una epistemología positivista y se desarrolla fundamentalmente en base a análisis muy sofisticados de variables 
sociolingüísticas. Si bien echan mano de factores sociales externos para explicarlo, su objetivo epistemológico último es la forma del lenguaje (Del Valle y Alvis 2017: 2).

No obstante, reconoce que

[e]l variacionismo en mi opinión fue muy importante en el momento en que emergió y durante un periodo significativo, fue una dialectología social altamente sofisticada desde el punto de vista teórico y también desde el punto de vista metodológico; entonces el variacionismo en la época original, en la época que asociamos con el trabajo de William Labov, de Peter Trudgill, lleva a los estudios de cambio lingüístico, por un lado, y los estudios de dialectología social, por otro, a un lugar de alta sofisticación teórica y metodológica, y además tiene una fuerte impronta política, en la medida en que uno de los efectos de la investigación variacionista es argumentar que distintas variedades de una lengua, distintas lenguas, tienen todas el mismo grado de complejidad estructural, o al menos el potencial para tener el mismo grado de complejidad estructural, para funcionar en cualquier contexto. No hay que olvidar nunca el trabajo de William Labov en nombre del inglés afroamericano vernáculo (Del Valle y Alvis 2017: 2).

La abstracción de los hablantes choca frontalmente con los intereses de la sociolingüística crítica. En este sentido, así podríamos ver la diferencia entre la sociolingüística ya clásica-la llamaremos aquí así: sociolingüística clásica, tal y como hace Pujolar (2012: 6), para diferenciarla de la "crítica"-y esta última, la sociolingüística crítica, en tanto que aquella centra su atención en aspectos lingüísticos, sin atender al contenido de lo que se dice ni las consecuencias de sus propias teorizaciones, al asumir categorías sociológicas sin problematizarlas, naturalizando relaciones de desigualdad; en cambio, la sociolingüística crítica, va más allá de ciertos aspectos lingüísticos descritos por la sociolingüística clásica a partir de categorías sociales asumidas, y centra su atención sobre el contenido de lo que transmiten los grupos sociales, sobre cómo se construyen las relaciones sociales y la imagen de la otredad a partir de los discursos o las características lingüísticas, percibidas como índices, del endo- y exogrupo (Pujolar 2012; Heller, Pujolar y Pietikäinen 2018). Por eso, también en Sociolingüítica crítica es importante la relación entre el poder y el lenguaje, como en el ACD o los ECD.

Desde esa perspectiva, el lenguaje y las lenguas son considerados procesos sociales y artefactos culturales, y deben ser analizados en relación con otras prácticas sociales y con otros artefactos culturales. Desentrañar las estrategias por medio de las cuales las prácticas 
lingüísticas o las representaciones de las lenguas son generadoras de desigualdad es fundamental para la sociolingüística crítica. Hay además un posicionamiento político que es definitorio de esa perspectiva (Del Valle y Alvis 2017: 3).

Si como ya hemos visto, la sociolingüística crítica estudia la estructuración social a través de la lengua, las jerarquizaciones sociales con la lengua como marcador y sus imbricaciones con otros marcadores de desigualdad, así como las ideologías lingüísticas, tanto los estudios de ACD desarrollados por Fairclough (1989), Wodak (1989), Martín Rojo (1997), Martín Rojo y van Dijk (1996), Wodak y Meyer (2001) o van Dijk (2009), como los trabajos sobre ideologías lingüísticas de Silverstein (1979, 1985, 2003), Irvine (1989), Gal (1989), Friedrich (1989) o Woolard y Schieffelin (1994), todos estos estudios, junto con las preocupaciones antiimperialistas de Calvet (1972), Phillipson (1991) o Moreno Cabrera (2000 y 2008), tienen en común lo crítico. Como sociolingüistas críticas y críticas pueden ser consideradas, entre otras autorías, Bloommaert (2010), Rampton (2006), Martín Rojo (2010), Pérez-Milans (2013), Patiño Santos (2018), Del Valle (2016), etc. En este sentido, la creación en 2014 de la Asociación de Estudios de Discurso y Sociedad (EDiSo), a parti de su primer simposio en Sevilla, supone un hito, auspiciada por Teun van Dijk y discípulas de John Gumperz y otras figuras históricas.

En Latinoamérica, una de las formas más potentes de crítica y establecimiento de lazos entre investigadoras universitarias diversas es la de la glotopolítica, con una mirada atenta a la naturaleza política del lenguaje. A partir de la destacada influencia de Elvira Narvaja de Arnoux en Argentina, en este país y en su vecino Chile se han desarrollado importantes trabajos y conformado puentes estratégicos de investigación. En Chile, destaca Darío Rojas y, en Argentina, un conjunto de investigadoras en torno a proyectos de primer orden: el Observatorio de Glotopolíticas de la Universidad Tres de Febrero de Buenos Aires, el grupo Lingüística Crítica de Universidad de Buenos Aires o el Círculo de Lingüística Feminista, así como el Centro de Estudios y Actualización en Pensamiento Político, Decolonialidad e Interculturalidad-CEAPEDI- de la Universidad Nacional del Comahue. Estas investigadoras son, además, de la propia Elvira Narvaja, Daniela Lauria, Diego Bentivegna, Ernesto Cuba, Mariana Di Stéfano, Gustavo Aprea, Roberto Bein, María Cecilia Pereira, entre otras muchas.

Las preocupaciones sobre el racismo lingüístico han tomado protagonismo en Perú, con Virginia Zavala, aunque cuenta con desarrollos previos como los de Rodolfo Marcial Cerrón-Palomino. En un sentido similar, en Chile, desde la filosofía, Patricio Lepe Carrión. Por su parte, a medio camino entre Nueva York y Lima, Myriam Yataco ha logrado reunir a investigadoras y 
activistas de todo el continente y lenguas en torno a su proyecto Derechos Lingüísticos.

Este es un panorama muy general que invita a abrir las puertas a publicaciones futuras sobre el conjunto de conocimientos y autorías que se están desarrollando en todo el continente en sentido crítico.

\section{A MODO DE CONCLUSIÓN}

Las condiciones sociales tras la llamada Segunda Guerra Mundial posibilitaron el nacimiento de la sociolingüística, acompañada del desarrollo de las ciencias sociales en campos como la psicología, la antropología o la sociología, que aportaron a los jóvenes lingüistas de finales de la década de 1950 y de principios de la década de 1960 conceptuaciones y metodologías con las que indagar y comprender la comunicación e interacción lingüística.

La mirada sobre las diferencias en cuanto el objeto (por muy positivista que esto pueda resultar) merece ser puesta en la brecha existente entre la sociolingüística clásica y la sociolingüística crítica. Si la primera está atravesada por el estructuralismo, en su concepción lingüística, la segunda "está anclada en los desarrollos teóricos y filosóficos asociados con la teoría crítica, sobre todo con la Escuela de Frankfurt" (Del Valle y Alvis 2017: 3). Sin embargo, en la primera, tal y como hemos apuntado, hay dos tendencias ( rosso modo), de tal forma que el proceder de Labov, en EE.UU., y el de Trudgill y Chambers (1980), en Inglaterra, pueden ser considerados los más estructuralista de la etapa clásica. Haciendo justicia a John Gumperz y Dell Hymes, hemos de reconocerlos como fuente o base, al interior de los estudios sociolingüísticos, para los desarrollos que hemos apuntado desde la década de 1980 en adelante.

Las ulteriores aportaciones del giro decolonial, completan las transformaciones epistemológicas que el posmodernismo -aun de base eurocéntrica-posibilitó a las ciencias sociales en general y a la sociolingüística en particular. Las experiencias desde las que conceptualizan los pensadores decoloniales, de lo que Fanon llamó la zona del no-Ser, han arrojado resultados interesantes en las dos primeras décadas del siglo XXI sobre las relaciones discursivas en los grupos subalternizados. Este giro decolonial se suma a aquel giro discursivo que coloca a la sociolingüística como una de las ciencias más necesarias del actual momento histórico: comprender lo que pasa a nuestro alrededor, en un mundo cambiante, globalizado y en 
conflicto permanente, pasa por comprender cómo interaccionamos y cómo conceptualizamos tanto las interacciones como a los demás grupos humanos, lo que por décadas hemos llamado con Gumperz comunidades de habla.

\section{REFERENCIAS BIBLIOGRÁFICAS}

Ammon, Ulrich, Norbert Dittmar y Klaus J. Mattheier (eds.). 1987-1988. Sociolinguistics. An International Handbook of the Science of Language and Society. Volumen 2. Berlín/ New York: Walter de Gruyter.

BLOCK, DAVID. 2013. Issues in language and identity research in applied linguistics. Estudios de lingüística inglesa aplicada ELIA 13: 11-46.

Blommaert, Jan. 2010. The Sociolinguistics of Globalization. Cambridge: Cambridge University Press.

2014. Sociolinguistics. En Constant Leung and Brian V. Street (eds.). The Routledge Handbook of English Language Studies, pp. 131-144. Londres: Routledge.

BorRego Nieto, Julio. 1981. Sociolingüistica rural. Investigación en Villadepera de Sayago. Salamanca: Universidad de Salamanca.

Bourdieu, Pierre. 1982. ¿Qué significar hablar? Economía de los intercambios lingüisticos. Madrid: Akal. Edición de 2008.

Calvet, Louis-Jean. 1972. Linguistique et colonialisme. Petit traité de glossophagie. Paris: Payot.

Cantera Ortiz de Urbina, Jesús. 1992. 'Lengua e imperio' en la política de la Revolución Francesa y sus antecedentes en la Península Ibérica en el siglo XVI. Revista de Filología Francesa 29 (1): 29-38.

Carbonero, Pedro. 2003. Estudios de Sociolingüística Andaluza. Sevilla: Secretariado de Publicaciones de la Universidad de Sevilla.

Casas Gómez, Miguel y Luis Escoriza Morera. 2009. Los conceptos de diastratía y diafasía desde la Teoría Lingüística y la Sociolingüística Variacionista. En María Victoria CamachoTaboada, José Javier Rodríguez Toro y Juana Santana Marrero (coord.). Estudios de lengua española: descripción, variación y uso: homenaje a Humberto López Morales, pp. 151-178. Madrid/Frankfurt am Main: Iberoamericana/Vervuert.

Cesaire, Aimé. 1950. Discurso sobre el colonialismo. Madrid: Akal. Edición de 2006.

Codó, Eva, Adriana PatiÑo y Virginia UnAmuno. 2012. Hacer sociolingüística etnográfica en un mundo cambiante: Retos y aportaciones desde la perspectiva hispana. Special issue of Spanish in Context 9 (2): 167-190.

Chomsky, Noam. 1957. Syntactic Structures. The Hague/París: Mouton. 1965. Aspects of the Theory of Syntax. Massachusetts: MIT.

Collins, Patricia Hill. 2000. Black feminist thought. Knowledge, consciousness, and the politics of empowerment. Nueva York: Routledge.

Coseriu, Eugenio. 1967. Teoría del lenguaje y Lingüistica general. Madrid: Gredos.

Coseriu, Eugenio. 1999. Lecciones de lingüistica general. Madrid: Gredos.

CURRIE, HAVER. 1952. A projection of sociolinguistics. The relationship of speech to social status. Southern Speech Journal 18: 28-37. 
Del Valle, José (ed.). 2016. Historia política del español. La creación de una lengua. Madrid: Editorial Aluvión.

Del Valle, José y Luis Gabriel-Stheeman (eds.). 2004. La batalla del idioma. La intelectualidad hispánica ante la lengua. Vervuert-Iberoamericana, Frankfurt/M.-Madrid.

Del Valle, José y Jorge Alvis. 2017. De la glotopolítica y la sociolingüística crítica a la racialización del español en los Estados Unidos. LL Journal 12 (1): 1-10.

Duranti, Alessandro. 2000. Antropología lingüística. Cambridge: University of Cambridge Press.

Dussel, EnRIQue. 1983. Praxis latinoamericana y filosofía de la liberación. Bogotá: Nueva América.

1996. Filosofía de la liberación. Bogotá: Nueva América.

FairClough, Norman. 1989. Language and power. Essex/Nueva York: Longman.

Fairclough, Norman y Ruth Wodak. 1997. Critical discourse analysis. En Teun van Dijk (ed.). Discourse Studies: A Multidisciplinary Introduction. Vol. 2, pp. 258-284. London: Sage.

FALS BORDA, OsWALDO. 1987. ¿Es posible una sociología de la liberación?. Ciencia propia y colonialismo intelectual. Los nuevos rumbos, pp. 15-23. Bogotá: Carlos Valencia Editores.

Fanon, Frantz. 1952. Peau noire masques blancs. Paris: Editions du Seuil.

1961. Los condenados de la Tierra. México: Fondo de Cultura Económica. Edición de 2001 .

Fernández, Mauro. 1996. Los orígenes de la Sociolingüística. Actas de las II Jornadas de Lingüistica, pp. 105-132. Cádiz: Servicio de Publicaciones de la Universidad de Cádiz.

FouCAUlt, Michel. 1975. Vigilar y castigar: el nacimiento de la prisión. Buenos Aires: Siglo XXI. Edición de 2002.

1979. Microfisica del poder. Madrid: Ediciones La Piqueta.

2003. Hay que defender la sociedad. Curso del Collège de France (1975-1976). Madrid: Akal.

Fowler, Roger (ed.). 1991. Language in the news. Discourse and ideology in the press. Londres: Toutledge.

Fowler, Roger, Bob Hodge, Gunther Kress y Tony Trew. 1979. Language and Control. Londres/Boston/Henley: Routledge/Kegan Paul.

FrIEDRICH, PAUl. 1989. Language, ideology, and political economy. American Anthropologist 91: 295-312.

GabelentZ, Georg von. 1891. Die Sprachwissenschaft. Ihre Aufgaben, Methoden und bisheringen Ergebnisse. Leipzig: T.O. Weigel.

Gal, Susan. 1989. Language and Political Economy. Annual Review of Anthropology 18 (1): 345-367.

García Marcos, Francisco. 2015. Sociolingüística. Madrid: Síntesis.

Grosfoguel, Ramón. 2007. Los dilemas de los estudios étnicos estadounidenses: multiculturalismo identitario, colonización disciplinaria y epistemologías decoloniales. Universitas Humanistica 63: 35-47.

2013. Racismo/sexismo epistémico, universidades occidentalizadas y los cuatro genocidios/epistemicidios del largo siglo XVI. Tabula Rasa 19: 31-58.

2018. ¿Negros marxistas o marxismos negros?: una mirada descolonial, Tabula Rasa 28: 11-22.

Heller, Monica, Sari PietikäInen y Joan Pujolar. 2018. Critical Sociolinguistic Research Methods. Studying Language Issues That Matter. Nueva York: Routledge.

Heller, Monica y Bonnie McElhinny. 2017. Language, capitalism, colonialism. Toward a Critical History. Toronto: University of Toronto Press. 
Hudson, R. A. 1981. La sociolingüística. Barcelona: Anagrama.

Hymes, Dell. 1967. Why Linguistics Needs the Sociologist. Social Research 34: 632-647.

Irvine, Judith T. 1989. When Talk Isn't Cheap: Language and Political Economy. American Ethnologist 16 (2): 248-267.

KoERnER, ERnST FrideryK KonRAD. 1986. Aux sources de la sociolinguistique. Lingvisticae Investigationes 10: 381-401.

1991. Toward a History of Modern Sociolinguistics. American Speech 66 (1): 57-70.

LABOv, William. 1966. The social stratification of English in New York City. Washington DC: Center for Applied Linguistics.

1983. Modelos sociolingüísticos. Madrid: Cátedra.

LeHMAN, Winfred P. 1981. Historical Linguistics and Sociolinguitics. International Journal of the Sociology of Language 31: 11-27.

Maldonado-Torres, Nelson. 2015. Transdisciplinariedad y decolonialidad. Quaderna 3.

Malkiel, Yakov. 1976. From Romance Philology through Dialect Geography to Sociolinguistics. International Journal of the Sociology of Language 9: 59-84.

Martín Rojo, Luisa. 1997. El orden social del discurso. Discurso 1: 1-37.

2010. Constructing Inequality in Multilingual Classrooms. Berlín/Nueva York: De Gruyter Mouton.

Martín Rojo, Luisa y Teun van Dijk. 1998. Había un problema y se ha solucionado: legitimación de la expulsión de inmigrantes "ilegales" en el discurso parlamentario español. En Luisa Martín Rojo y Rachel Whittaker (coords.). Poder-decir: o el poder de los discursos, pp. 169-234. Madrid: Arrecife.

Moreno Cabrera, Juan Carlos. 2000. La dignidad e igualdad de las lenguas. Crítica de la discriminación lingüistica. Madrid: Alianza.

2008. El nacionalismo lingüistico. Una ideología destructiva. Barcelona: Península.

Murray, Stephen O. 1983. Group Formation in Social Science. Carbondale/Edmonton: Linguistic Research.

1994. Theory Groups and the Study of Language in North America: A Social History. Amsterdam: John Benjamins.

Patino Santos, Adriana. 2018. No-one told me it would all be in Catalan! Narratives and language ideologies in the Latin American community at school. International Journal of the Sociology of Language 250: 59-86.

Pérez-Milans, Miguel. 2012. Ah! Spain, that's far away from China: Reflexividad metodológica y movilidad en la etnografía sociolingüística crítica. Spanish in Context 9 (2): 219-243.

2013. Urban Schools and English Language Education in Late Modern China: A Critical Sociolinguistic Ethnography. Nueva York: Routledge.

Phillipson, Robert. 1992. Linguistic Imperialism. Oxford: Oxford University Press.

Pujolar, JoAn. 2012. La sociolingüística crítica. En Maite Puigdevall, Raquel Casesnoves, Josep Conill, Eva Codó y Joan Pujolar (eds.). Sociolingüistica, pp. 1-58. Barcelona: Editorial UOC.

Rampton, Ben. 2006. Language in Late Modernity. Interaction in an Urban School. Cambridge: Cambridge University Press.

Rodney, Walter. 1972. How Europe Underdeveloped Africa. Dar es Salaam/London: Tanzania Publishing House/Bogle L'Ouverture Publications.

RODRÍGUEZ-IGLESIAS, ÍGOR. 2013. Generalización diastrática y restricción diafásica en variación diatópica. En Adrián Cabedo Nebot, Manuel Jesús Aguilar Ruiz y Elena LópezNavarro Vidal (ed.). Estudios de lingüística: investigaciones, propuestas y aplicaciones, pp. 581-593. Valencia: Universitat de València. 
2016. Ideologías lingüísticas: descapitalización fanoniana de los andaluces. Nueva Revista del Pacifico 65: 105-136.

2017. Interseccionalidad en una heterojerarquía de dominación: mi mujer/su mujer. En Juan Carlos Suárez Villegas, Luis Alfonso Guadarrama Rico, Jannet Valero Vilchis y Paola Panarese (eds.). La comunicación de género invisibilizada en la comunicación, pp. 75-87. Madrid: Dykinson.

2018a. La relación entre acto intencional, significación e intuición complementadora husserlianos y los niveles del significar semánticos. e-Scripta Romanica 6: 65-76.

2018b. La ideología lingüística de Juan Valdés sobre el andaluz desde una perspectiva decolonial. Hito en la construcción del privilegio lingüístico. Circula: revue d'idéologies linguistiques 7: 90-114.

Romaine, SuZANNE. 1994. Language in society: An introduction to sociolinguistics. Oxford/ New York: Oxford University Press.

RuesCh, Jurgen. 1951. Values, Communication, and Culture: An Introduction. En J. Ruesch y G. Bateson. Communication. The Social Matrix of Psychiatry, pp. 3-20. Nueva York: W. W. Norton and Company.

SAPIR, EdWARD. 1938. Why cultural anthropology needs the psychiatrist. Psychiatry. Interpersonal and Biological Processes 1 (1): 7-12.

Schwendinger, Herman, Julia R. Schwendinger y Michael J. Lynch. 2002. Critical Criminology in the United States: The Berkley School and Theoretical Trajectories. En Kerry Carrington y Russell Hogg (eds.). Critical Criminology: Issues, Debates, Challenges, pp. 41-72. Devon/Portland: Willan Publishing.

SpOlSKY, Bernard. 2011. Ferguson and fishman: Sociolinguistics and the sociology of language. En Ruth Wodak, Barbara Johnstone y Paul Kerswill (eds.). The SAGE handbook of sociolinguistics, pp. 11-24. London: SAGE.

van Dijk, Teun. 2009. Discurso y poder. Barcelona, Gedisa.

van Dijk, Teun A. 2015. Cincuenta años de estudios del discurso. Discurso \& Sociedad 9 (1-2): 15-32.

WodAK, Ruth. 2003. El enfoque histórico del discurso. En Ruth Wodak y Michael Meyer (eds.). Métodos de análisis crítico del discurso, pp.101-142. Barcelona: Gedisa.

Yule, George. 2007. El lenguaje. Cambridge: Cambridge University Press. 